\title{
Tertiary hospitals must provide general care
}

\author{
Ken Flegel MDCM MSc
}

$\mathrm{T}$ he term "tertiary" may be a useful way to categorize a university hospital or specialist centre, but when the term is used to describe the care given to a patient, it is a vacuous construct. When hospital administrators define some patients or medical conditions as outside the remit of tertiary care, doctors come under pressure to think in silos and to abandon their patients.

The most important problem with the idea of "tertiary medical care" is that it is focused on the disease rather than on, as William Osler might say, the person who has the disease. This lapse would be merely laughable if it were a simple oversight; however, it is potentially more serious than that. Not all patients in tertiary centres will fit into a particular eligibility profile, but they nevertheless may have reasons for needing highly knowledgeable and skilled care. The need for care in a tertiary setting may arise not from the rarity or complexity of the condition, but from multimorbidity or a combination of a person's outlook, personality, and personal and family circumstances. Although the condition itself may be relatively straightforward, managing it may require all the knowledge, skill, authority or resources of the subspecialist. Conversely, the fact that a patient is receiving care in a tertiary centre should not restrict the medical care given to only complex specialist care, but should encompass the care of the whole person.

The second problem is that the term implies that we generally care for a patient by working up through the three levels of medical care (primary through tertiary). However, there are types of care for which proceeding ordinally would be simply stupid or potentially dangerous; the patient with severe or multiple trauma, or the patient requiring an organ transplant would be examples. In the first case, providing primary care could cause fatal delay; tertiary care should come first. In the second case, many people need a transplant, but only a minority receives one. Where needed care is not accessible, it obviously cannot be called tertiary care in this ordinal sense.

The term tertiary care came into hospital parlance a few decades ago, but only in 2013 was it integrated into the US National Library of Medicine's Medical Subject Headings thesaurus, when it was defined as "care of a highly technical and specialized nature, provided in a medical center, usually one affiliated with a university, for patients with unusually severe, complex, or uncommon health problems." A PubMed search on "tertiary care" will yield 40000 papers, which mostly use the expression to highlight the specialized nature of the authors' knowledge, technology or treatment. Typical examples of tertiary care are severe and multiple trauma, organ transplant, dynamic hormone testing and transcatheter heart-valve implantation. The common thread is the esoteric nature of the expertise.

That omnibus definition is problematic in that the determination of what aspects of medical care are or are not included is in the mind of those providing the care. Obviously, there are funding implications for the academic institutions that have this label, and prestige and billing benefits for the practitioners, but tertiary hospitals must continue to provide general medical and surgical services if they are to provide high-quality subspecialist care, as well.

To the extent that a tertiary care hospital espouses special levels of care to the exclusion of all others, it risks becoming obsolete and dangerous, because it severs itself from the wellspring of inspiration, innovation and correction that hospital medical practice truly exemplifies. Such a hospital will likely not be able to meet the broad training needs of future practitioners. Moreover, standard-setting and surveillance require the vigilant participation of a coherent and broadly based profession. And to practise exclusive care is to ignore the hospital care needs of the local community. Great hospitals do not make this mistake.

Classifying health care by level of severity and complexity is probably useful to the third-party payer and the health care administrator, but it makes little sense to the practitioner who must advocate for the needs of the particular patient. Modern medical practice is blessed with many diagnostic and therapeutic options for any illness predicament. Medical practice proceeds one patient at a time and not by bureaucratic levels of care or by eligibility; it ought to offer every specific opportunity for benefit that the physician can imagine and execute. It is unacceptable for a practitioner to be prevented from pursuing the one most likely to benefit a particular patient simply because some bureaucrat or academic has classified the medical practice as tertiary care. If we allow patient care in our academic hospitals to be divided in a way that makes no sense for the patient, we shall be ignoring Francis W. Peabody's famous admonition, "the secret of the care of the patient is in caring for the patient." If we fail to do this, we may not be the ones who direct that medical care for much longer.

\section{References}

1. National Center for Biotechnology Information. Tertiary healthcare. Available: www.ncbi.nlm.nih.gov/mesh/68063128 (accessed 2014 Jan. 14).

2. Peabody FW. The care of the patient. Cambridge (MA): Harvard University Press; 1927:48.

Competing interests: See www.cmaj.ca/site/misc/cmaj_staff.xhtml

Affiliation: Senior Editor, CMAJ.

Correspondence to: $C M A J$ editor, pubs@cmaj.ca

CMAJ 2015. DOI:10.1503/cmaj.150056 